\title{
Adult medulloblastoma: Multiagent chemotherapy
}

\author{
Harry S. Greenberg, ${ }^{1}$ Marc C. Chamberlain, Michael J. Glantz, and Songbai Wang \\ Departments of Neurology (H.S.G.) and Biostatistics (S.W.), University of Michigan, Ann Arbor, MI 48109; \\ Department of Neurology, Kaiser-Permanente, Baldwin Park, CA 91706 (M.C.C.); Department of Neurology, \\ Brown University, Providence, RI 02912 (M.J.G.)
}

In this study, the records of 17 adult patients with medulloblastoma treated with craniospinal radiation and 1 of 2 multiagent chemotherapy protocols were reviewed for progression-free survival, overall survival, and toxicity, and the patients were compared with each other and with similarly treated children and adults. Records of patients treated at 3 institutions were reviewed. Seventeen medulloblastoma patients ( 11 female, 6 male) with a median age of 23 years (range, 18-47 years) were treated with surgery, craniospinal radiation (CSRT) plus local boost, and 1 of 2 adjuvant chemotherapy regimens. All tumors were infratentorial (10 in 4th ventricle and 7 in left or right hemisphere). Ten patients presented with hydrocephalus, and 7 of them were shunted. Eight patients had gross total resection, 7 had subtotal resection ( $>50 \%$ removed), and 2 had partial resection ( $<50 \%$ removed). Postoperatively, 3 patients had positive cytology and 3 had positive spinal MRI. Five patients were classified as good risk and 12 were classified as poor risk (Chang staging system). Ten patients were treated with the "Packer protocol," consisting of CSRT plus weekly vincristine followed by 8 cycles of cisplatin, lomustine, and vincristine. Seven patients were treated with the Pediatric Oncology Group (POG) protocol, consisting of alternating courses of cisplatin/etoposide and cyclophosphamide/vincristine, followed by CSRT. Eight of 17 patients relapsed, with all 8 relapsing at the primary site. Other relapse sites included the leptomeninges (5), bone (1), and brain (1). The estimated median relapse-free survival (Kaplan-Meier) for all patients was 48 months $(95 \%$ confidence interval, $>26$ months to $\infty)$. Median relapse-free survival for patients on the Packer protocol was 26 months, and for those on the POG regimen was

Received 1 March 2000, accepted 23 May 2000.

\footnotetext{
${ }^{1}$ Address correspondence and reprint requests to H.S. Greenberg, Department of Neurology, University of Michigan, 1914 Taubman Center, Box 0316, Ann Arbor, MI 48109.
}

${ }^{2}$ Abbreviations used are as follows: CCNU, lomustine; CDP, cisplatin; CTX, cyclophosphamide; POG, Pediatric Oncology Group; VCR, vincristine.
48 months $(P=0.410)$. Five of 10 on the Packer protocol were relapse-free, while 4 of 7 were relapse-free on the POG regimen. Two patients relapsed during chemotherapy and 6 relapsed after completing all therapy at 18 , $18,26,30,40$, and 48 months. The estimated median survival of all patients was 56 months $(95 \%$ confidence interval, 27 to $\infty$ ) with 11 patients alive; for the Packer protocol, median survival was 36 months, and for the POG protocol, it was 57 months $(P=0.058)$. The hazard ratio was $0(95 \%$ confidence interval, 0 to $\infty)$. Toxicity during the Packer protocol was moderately severe, with only 1 of 10 patients able to complete all therapy. Two patients had severe abdominal pain during CSRT + vincristine, and 5 had peripheral neuropathy during vincristine therapy. Hearing loss $(>20 \mathrm{~dB})$ occurred in 7 , neutropenia $(<500 \mu \mathrm{l})$ in 6 , thrombocytopenia $(<50,000$ $\mu \mathrm{l})$ in 6 , nephrotoxicity ( $>25 \% \downarrow$ by creatinine clearance) in 2 , and decreased pulmonary function (diffusing capacity for carbon monoxide $\downarrow>40 \%$ ) in 1 . On the POG protocol, only 1 patient had persistent nausea and vomiting, 2 had peripheral neuropathy, and 3 had hearing deficit $(>20 \mathrm{~dB})$ or tinnitus. The POG and Packer protocols did not have a statistically significant difference in relapsefree or overall survival because of the small sample size. The POG protocol seemed to have less nonhematologic toxicity. Adults on the Packer protocol appeared to have shorter median survival and greater toxicity than did children. To know whether adding adjuvant chemotherapy to craniospinal radiation in adult therapy increases relapse-free and overall survival, we must await the results of a larger randomized controlled clinical trial. Neuro-Oncology 3, 29-34, 2001 (Posted to NeuroOncology [serial online], Doc. 00-014, October 3, 2000. $U R L<$ neuro-oncology.mc.duke.edu>)

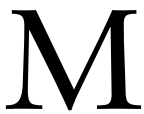
edulloblastoma is the most common CNS tumor of childhood, accounting for $15 \%$ to $30 \%$ of all childhood brain tumors, and $30 \%$ to $40 \%$ of all posterior fossa tumors (Hubbard et al., 1989). The tumor occurs with a peak incidence in the first decade of life, with a bimodal distribution peaking at 3 to 4 years of age and again at 8 to 9 years. In adults, the tumor is much less common, accounting for approxi- 
mately $1 \%$ of adult primary brain tumors, $80 \%$ of which occur before the end of the fourth decade (Aragones et al., 1994; Bloom et al., 1990; Kopelson et al., 1982). Medulloblastoma is thought to arise from a primitive cell in the external granular layer of the cerebellum, but the specific cell of origin has not been identified definitively. The adult tumor is said to occur more often in the cerebellar hemispheres than the vermis and is more often desmoplastic in histology (Aragones et al., 1994; Frost et al., 1995; Prados et al., 1995). Karyotypic analysis of medulloblastoma finds frequent structural abnormalities, with chromosome 17 the most frequently affected (Biegel et al., 1989).

High-risk childhood medulloblastomas have been treated with maximal surgery, craniospinal radiation therapy, and adjuvant chemotherapy for the last 10 years (Packer et al., 1991, 1994). A multiagent chemotherapy protocol-CDP, ${ }^{2}$ CCNU, and VCR - has increased the 5 -year progression-free survival rate in high-risk children to $85 \%$ (Packer et al., 1994). Survival of high-risk patients was better than that of low-risk patients treated by radiation alone (Packer et al., 1991, 1994). Therefore, we decided to treat both good- and poor-risk adult patients with craniospinal radiation and multiagent therapy (Le et al., 1997; Prados et al., 1995). Relapse occurs predominately at the original site, but also in the leptomeninges and extraneurally in bone (Packer et al., 1985; Prados et al., 1995).

We reviewed our institutions' experiences treating 17 adult patients who had medulloblastoma and who were treated with maximal surgery, craniospinal radiation therapy, and 1 of 2 multiagent chemotherapy regimens. In this study we report our overall results and compare treatment outcomes for each of the 2 regimens. We also contrast our experience using craniospinal radiation and chemotherapy with the results obtained in the pediatric population and in adults treated with radiation therapy alone.

\section{Materials and Methods}

We performed a retrospective review of the charts of 17 consecutive patients who had infratentorial medulloblastoma, were aged 18 years or older, and were treated at diagnosis with surgery, craniospinal radiation therapy, and multiagent chemotherapy. Patients diagnosed between 1991 and 1997 were accrued from 3 institutions: University of Michigan, University of California at San Diego, and Brown University. They were treated at the University of Michigan and the University of California at San Diego on the Packer protocol (described below) and at Brown University on both the Packer and the POG protocols (described below) without randomization. Patients were staged according to Chang criteria (Chang et al., 1969) with spinal neuraxis MRI and lumbar puncture including cytology. According to Chang criteria, a rating $>\mathrm{T} 2$ and/or $>\mathrm{M} 0$ was considered poor risk. Data were tabulated on a specially developed worksheet. All patients were given craniospinal radiation therapy, with 15 of 17 treated at a dose of $36 \mathrm{~Gy} ; 1$ patient on the POG protocol was treated at $25.5 \mathrm{~Gy}$, and 1 was treated at $45.5 \mathrm{~Gy}$. The posterior fossa of all patients received a boost of 53.2 to $60 \mathrm{~Gy}$ (median $54 \mathrm{~Gy}$ ). One of 2 chemotherapy regimens was used to treat all patients as follows: (1) weekly VCR $\left(1.5 \mathrm{mg} / \mathrm{m}^{2}\right)$ during craniospinal radiation therapy and for 6 weeks thereafter; then 8 cycles ( 1 cycle every 6 to 8 weeks) of CDP $\left(75 \mathrm{mg} / \mathrm{m}^{2}\right)$ and CCNU $\left(50 \mathrm{mg} / \mathrm{m}^{2}\right)$ on day 1 and VCR $\left(1.5 \mathrm{mg} / \mathrm{m}^{2}\right)$ given 3 times per cycle. Ten patients were treated with this regimen based on a protocol designed by Packer et al. $(1985,1991)$. (2) Preradiation chemotherapy with cycles of CDP $\left(20 \mathrm{mg} / \mathrm{m}^{2}\right.$ on days $1-5$ or $90 \mathrm{mg} / \mathrm{m}^{2}$ on day 1) and etoposide $(75-100$ $\mathrm{mg} / \mathrm{m}^{2}$ for 3 to 5 days) alternating with CTX (450-1580 $\mathrm{mg} / \mathrm{m}^{2}$ on days 1 and 2$)$ and VCR $\left(1.5 \mathrm{mg} / \mathrm{m}^{2}\right.$ on day 1$)$ (Duffner et al., 1993). Seven patients were treated on this regimen based on a protocol designed by POG (Duffner et al., 1993).

Estimated median time to relapse and survival time were calculated using Kaplan-Meier analysis. Comparisons of relapse-free survival, survival by treatment group, and survival by risk group were performed using an exact version of the log-rank test. A hazard ratio for relapse and overall survival was calculated between treatment groups and risk groups.

Response was defined according to MacDonald criteria (MacDonald et al., 1990). Relapse was statistically defined as a $25 \%$ increase in contrast-enhancing tumor at the original site, as identification of a new site of disease, or as the patient's death if relapse had not occurred.

\section{Results}

\section{Risk and Treatment}

There were 6 male and 11 female patients in this study (Table 1). The median age was 23 years (range, 18-47 years). In 10 patients, the tumor was in the vermis or fourth ventricle, and in 7 it was in the cerebellar hemispheres ( 5 right, 2 left). Twelve patients were poor-risk patients according the Chang staging system. Seven of these were T3, 2 of whom had CSF spread on lumbar puncture; 1 was M1, and 1 was M3. Five additional patients had metastatic disease (M2-M4). Six of the 12 poor-risk patients were treated on the Packer protocol $(60 \%)$, and 6 were treated on the POG protocol $(84 \%)$. The difference in the number of poor-risk patients on each protocol was not statistically significant. Patients were treated with partial $(<50 \%)$ resection $(2)$, subtotal $(>50 \%)$ resection (7), and gross total resection (8), as defined by postoperative contrast-enhanced cranial MRI. Postoperatively, 3 patients had positive cytology, 2 at greater than 3 weeks postoperatively. Three patients had spinal MRIs positive for disseminated disease; 1 of these 3 had nodular disease and was the third patient with a positive cytology 2 weeks postoperatively. Seven patients had hydrocephalus and underwent ventriculoperitoneal shunt placement. Three patients had hydrocephalus and did not undergo shunt placement. Three patients had shunt placement without 


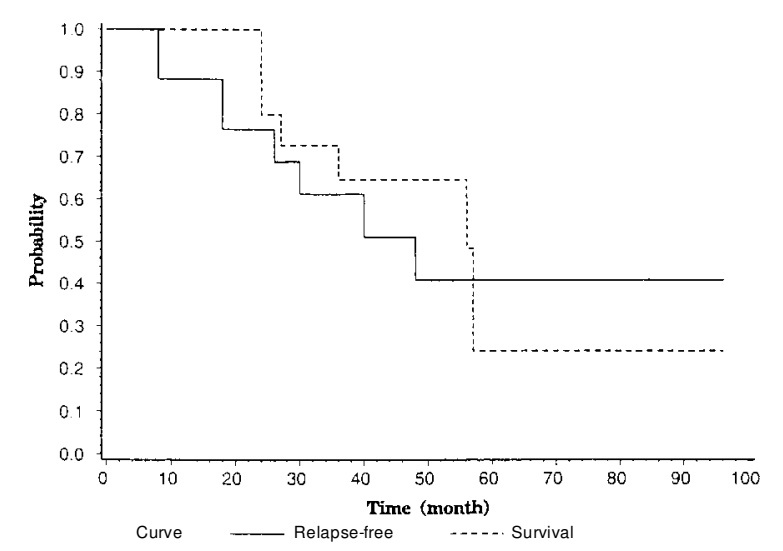

Fig. 1. Overall survival and progression free curves for all 17 patients treated.

hydrocephalus, and 4 had neither hydrocephalus nor shunt procedure.

\section{Relapse-Free and Overall Survival}

The Kaplan-Meier estimated median relapse-free survival for all patients was 48 months $(95 \%$ confidence interval, 26 to $\infty$ ), with 8 patients having relapsed (Fig. 1). Ten patients ( 5 relapses) treated on the Packer protocol were estimated to have a median 26-month relapse-free survival $(95 \%$ confidence interval, 18 to $\infty)$; the 7 patients ( 3 relapses) treated on the POG protocol had an estimated median 48-month relapse-free survival (95\% confidence interval, 30 to $\infty)(P=0.410)$. The hazard ratio was 0.59 (95\% confidence interval, 0.14-2.52). Relapses were local (at the site of the intracranial tumor) in 8 patients: leptomeninges (5), bone (1), and brain (1). The KaplanMeier estimated median overall survival was 56 months (95\% confidence interval, 27 to $\infty$ ) (Fig. 1). The 10 patients treated on the Packer protocol were estimated to have 36 months of overall median survival (95\% confidence interval, 24 to $\infty$ ), and the 7 POG protocol patients were estimated to have 57 month of overall median survival $(95 \%$ confidence interval, 56 to $\infty, P=0.058)$. The hazard ratio for overall survival was $0(95 \%$ confidence interval, 0.0 to $\infty$ ). When Chang criteria were used to classify patients as good or poor risk, there was no difference in relapse-free survival $(P=0.570)$ or overall survival $(P=$ 0.161 ) in the 2 groups. The hazard ratio was not significant at 0.66 (95\% confidence interval, $0.16-2.80)$ for relapse-free survival or for overall survival at $0.32(95 \%$ confidence interval, 0.06-1.58). There was also no difference in relapse-free or overall survival by risk group according to treatment, and no difference in relapse-free $(P=0.136)$ or overall survival $(P=0.258)$ whether primary location was in the cerebellar hemispheres or in other posterior fossa locations.

\section{Packer Protocol}

Ten patients were treated on the Packer protocol (Table 2 ). Seven patients had a gross total resection and were
Table 1. Baseline demographics of patients by treatment protocol

\begin{tabular}{lcc}
\hline & Packer $^{\mathrm{a}}$ & POG $^{\mathrm{b}}$ \\
\hline Age & 24.1 & 29.6 \\
Mean & 21.5 & 29 \\
Median & $18-34$ & $21-47$ \\
Range & $4 / 6$ & $2 / 5$ \\
Sex (M/F) & & \\
Disease Staging & $4(40 \%)$ & $1(16 \%)$ \\
Good Risk & $6(60 \%)$ & $6(84 \%)$ \\
Poor Risk & & $1(16 \%)$ \\
Resection & $7(70 \%)$ & $6(84 \%)$ \\
Complete & $3(30 \%)$ & \\
Partial &
\end{tabular}

Abbreviation: POG, Pediatric Oncology Group.

${ }^{a}$ Treatment protocol based on Packer et al., 1985, 1991.

${ }^{\text {b}}$ Treatment protocol based on Duffner et al., 1993.

stable throughout radiation and chemotherapy. Two patients had a subtotal resection $(>50 \%)$, and both had a complete response after radiation therapy. The patient who had a partial resection of $<50 \%$ had a complete response after radiation. Two patients progressed during chemotherapy at 8 and 9 months.

\section{POG Protocol}

Seven patients were treated on the POG protocol (Table 2 ). One patient had a gross total resection and remained stable throughout all treatment. Five patients had subtotal resection, 3 of whom had partial responses after 2 or 3 cycles of chemotherapy that became complete responses after radiation. One patient was stable throughout chemotherapy and radiation. One patient progressed during chemotherapy and was stable during radiation. She is stable 25 months after diagnosis. One patient had a partial resection and was stable throughout radiation and chemotherapy. The 5 relapsed patients on the Packer protocol survived a median of 10 months (6, $9,10,13$, and 16 months) after relapse, and the 3 relapsed patients on the POG protocol survived a median of 8 months $(4,8$, and 24 months).

\section{Chemotherapy Complications}

Complications of the 2 chemotherapy regimens are listed in Table 3. Seven of the 10 patients were able to complete the planned 8 cycles of the Packer protocol; 1 patient completed 6 cycles, and 2 patients, 4 cycles. Nine of the 10 patients required dose reduction or discontinuation of all 3 drugs. Cisplatin dosages were decreased in 8 patients after 3 to 6 cycles and were changed to carboplatin in 2 patients because of nephrotoxicity and ototoxicty. CCNU dose was decreased in 10 patients after 1 to 6 cycles because of myelosuppression. Also on the Packer proto$\mathrm{col}$, neutropenia $<500 \mu \mathrm{l}$ was seen in 6 patients during 20 cycles of chemotherapy. Thrombocytopenia $<50,000 \mu \mathrm{l}$ was seen in 6 patients during 20 cycles of chemotherapy. Anemia $<10 \mathrm{~g}$ hemoglobin was seen in 3 patients on 14 
H.S. Greenberg et al.: Adult medulloblastoma chemotherapy

Table 2. Demographics, tumor location, staging, and treatment outcome of patients on 2 protocols studied

\begin{tabular}{|c|c|c|c|c|c|c|c|c|c|}
\hline Patient & Age & Sex & $\begin{array}{l}\text { Tumor location } \\
\text { \& surgery }\end{array}$ & $\begin{array}{l}\text { Chang } \\
\text { staging }\end{array}$ & $\begin{array}{l}\text { Relapse-free } \\
\text { survival (mo.) }\end{array}$ & $\begin{array}{l}\text { Relapse } \\
\text { site }\end{array}$ & $\begin{array}{c}\text { Overall } \\
\text { survival (mo.) }\end{array}$ & $\begin{array}{l}\text { Chemotherapy } \\
\text { toxicity }^{\mathrm{a}}\end{array}$ & $\begin{array}{l}\text { Chemotherapy } \\
\text { dose modification }\end{array}$ \\
\hline \multicolumn{10}{|l|}{$\begin{array}{l}\text { Packer } \\
\text { protocol }\end{array}$} \\
\hline 1 & 18 & M & $\begin{array}{l}\text { Hydrocephalus } \\
\text { R cerebellum } \\
\text { GTR }\end{array}$ & $\begin{array}{l}\text { M0 } \\
\text { T3a } \\
\text { Poor }\end{array}$ & $39+$ & - & $39+$ & $\begin{array}{l}\downarrow \text { Creatinine } \\
\quad \mathrm{cl}(4)\end{array}$ & $\begin{array}{c}\Delta \text { To carboplatin } \\
\text { after } 4 \text { courses } \\
\downarrow \text { CCNU dose }\end{array}$ \\
\hline 2 & 33 & $F$ & $\begin{array}{l}\text { Hydrocephalus } \\
\text { L cerebellum } \\
\text { GTR }\end{array}$ & $\begin{array}{c}\text { Mo } \\
\text { T2 } \\
\text { Good }\end{array}$ & $23+$ & - & $23+$ & $\begin{array}{l}\text { Hearing loss (4) } \\
\text { Neutropenia (1) } \\
\text { Abd. pain, nausea, } \\
\text { \& vomiting (3) }\end{array}$ & $\begin{array}{c}\text { Only } 4 \text { courses } \\
\text { chemotherapy } \\
\downarrow \text { CCNU dose } \\
\downarrow \text { VCR dose all courses }\end{array}$ \\
\hline 3 & 27 & $\mathrm{~F}$ & $\begin{array}{l}\text { Vermis } \\
\text { STR }\end{array}$ & $\begin{array}{l}\text { M0 } \\
\text { T3a } \\
\text { Poor }\end{array}$ & $31+$ & - & $31+$ & $\begin{array}{c}\text { Hearing loss (5) } \\
\downarrow \text { Creatinine cl (5) } \\
\text { Lung fibrosis (6) } \\
\text { Thrombocytopenia (1) } \\
\text { Abd. pain } \\
\text { (during RT/VCR) }\end{array}$ & $\begin{array}{c}\Delta \text { To carboplatin } \\
\text { after } 6 \text { courses } \\
\text { D/C CCNU } \\
\text { after } 6 \text { courses }\end{array}$ \\
\hline 4 & 23 & M & $\begin{array}{l}\text { Vermis } \\
\text { GTR }\end{array}$ & $\begin{array}{c}\text { M0 } \\
\text { T1 } \\
\text { Good }\end{array}$ & $55+$ & - & $55+$ & $\begin{array}{c}\text { Hearing loss (4) } \\
\text { Abd. pain } \\
\text { (during RT/VCR) } \\
\text { Autonomic } \\
\text { neuropathy } \\
\text { (during RT/VCR) }\end{array}$ & $\begin{array}{c}\text { Only } 6 \text { courses } \\
\text { chemotherapy } \\
\text { no VCR during } \\
\text { chemotherapy } \\
\downarrow \text { CCNU after } 4 \text { courses }\end{array}$ \\
\hline 5 & 19 & M & $\begin{array}{c}\text { 4th Ventricle } \\
\text { GTR }\end{array}$ & $\begin{array}{l}\text { M0 } \\
\text { T3 } \\
\text { Poor }\end{array}$ & 26 & $\begin{array}{c}\text { Primary } \\
\text { leptomeningeal }\end{array}$ & 36 & $\begin{array}{l}\text { Hearing loss (6) } \\
\text { Neutropenia (6) } \\
\text { Peripheral } \\
\text { neuropathy (1) }\end{array}$ & $\begin{array}{c}\downarrow \text { CDP dose } \\
\downarrow \text { CCNU dose } \\
\text { D/C VCR } \\
\text { after } 3 \text { courses }\end{array}$ \\
\hline 6 & 34 & M & $\begin{array}{l}\text { L cerebellum } \\
\text { STR }\end{array}$ & $\begin{array}{l}\text { M0 } \\
\text { T3 } \\
\text { Poor }\end{array}$ & 18 & $\begin{array}{c}\text { Primary } \\
\text { leptomeningeal }\end{array}$ & 27 & $\begin{array}{c}\text { Hearing loss (6) } \\
\text { Thrombocytopenia (5) } \\
\text { Neutropenia (4) } \\
\text { Peripheral } \\
\text { neuropathy (1) }\end{array}$ & $\begin{array}{c}\downarrow \text { CDP dose } \\
\downarrow \text { CCNU dose } \\
\text { D/C VCR } \\
\text { after } 4 \text { courses }\end{array}$ \\
\hline 7 & 19 & $\mathrm{~F}$ & $\begin{array}{l}\text { 4th Ventricle } \\
\text { GTR }\end{array}$ & $\begin{array}{l}\text { M0 } \\
\text { T3 } \\
\text { Poor }\end{array}$ & $48+$ & - & $48+$ & $\begin{array}{c}\text { Thromobocytopenia (6) } \\
\text { Neutropenia (6) } \\
\text { Peripheral } \\
\text { neuropathy (4) }\end{array}$ & $\begin{array}{c}\downarrow C D P \& C C N U \\
\text { dose } \\
\text { only } 6 \text { courses VCR }\end{array}$ \\
\hline 8 & 20 & $\mathrm{~F}$ & $\begin{array}{l}\text { 4th Ventricle } \\
\text { PR }\end{array}$ & $\begin{array}{l}\text { M2 } \\
\text { T3 } \\
\text { Poor }\end{array}$ & 8 & $\begin{array}{c}\text { Primary } \\
\text { corpus callosum } \\
\text { leptomeningeal }\end{array}$ & 24 & $\begin{array}{c}\text { Hearing loss (7) } \\
\text { Thrombocytopenia (4) } \\
\text { Neutropenia (4) } \\
\text { Anemia (4) } \\
\text { Peripheral } \\
\text { neuropathy (1) }\end{array}$ & $\begin{array}{c}\downarrow \text { CDP dose } \\
\downarrow \text { CCNU dose } \\
\text { only } 3 \text { courses } \\
\text { vincristine }\end{array}$ \\
\hline 9 & 19 & $\mathrm{~F}$ & $\begin{array}{l}\text { R cerebellum } \\
\text { GTR }\end{array}$ & $\begin{array}{l}\text { M0 } \\
\text { T2 } \\
\text { Good }\end{array}$ & 9 & $\begin{array}{c}\text { Primary } \\
\text { leptomeningeal }\end{array}$ & 24 & $\begin{array}{c}\text { Hearing loss (7) } \\
\text { Anemia (4) } \\
\text { Neutropenia (4) } \\
\text { Thrombocytopenia (4) } \\
\text { peripheral } \\
\text { Neuropathy (1) }\end{array}$ & $\begin{array}{l}\downarrow \text { Cisplatin dose } \\
\downarrow \text { CCNU dose } \\
\text { only } 3 \text { courses } \\
\text { vincristine }\end{array}$ \\
\hline 10 & 29 & $\mathrm{~F}$ & $\begin{array}{l}\text { Hydrocephalus } \\
\text { R cerebellum } \\
\text { GTR }\end{array}$ & $\begin{array}{l}\text { Mo } \\
\text { T1 } \\
\text { Good }\end{array}$ & 18 & Primary & 24 & $\begin{array}{c}\text { Thrombocytopenia (1) } \\
\text { Anemia (1) }\end{array}$ & $\begin{array}{l}\text { Only } 4 \text { courses } \\
\text { chemotheraphy }\end{array}$ \\
\hline \multicolumn{10}{|l|}{ Protocol } \\
\hline 11 & 21 & $\mathrm{~F}$ & $\begin{array}{l}\text { Hydrocephalus } \\
\text { 4th Ventricle } \\
\text { STR }\end{array}$ & $\begin{array}{l}\text { M1 } \\
\text { T3 } \\
\text { Poor }\end{array}$ & $56+$ & - & $56+$ & $\begin{array}{c}\text { Tinnitus (1) } \\
\text { Absent } \\
\text { ankle jerks (2) }\end{array}$ & \\
\hline 12 & 47 & $\mathrm{~F}$ & $\begin{array}{l}\text { Hydrocephalus } \\
\text { Vermis } \\
\text { STR }\end{array}$ & $\begin{array}{l}\text { M0 } \\
\text { T1 } \\
\text { Good }\end{array}$ & 48 & Primary & 56 & & \\
\hline 13 & 29 & $\mathrm{~F}$ & $\begin{array}{l}\text { Hydrocephalus } \\
\text { R cerebellum } \\
\text { STR }\end{array}$ & $\begin{array}{l}\text { M1 } \\
\text { T1 } \\
\text { Poor }\end{array}$ & 30 & $\begin{array}{c}\text { Primary } \\
\text { leptomeningeal } \\
\text { bone }\end{array}$ & 57 & $\begin{array}{c}\text { Hearing loss } \\
(2) \\
\text { Neutropenia } \\
(1) \\
\text { Thromobocytopenia } \\
(1) \\
\text { Anemia (1) }\end{array}$ & $\downarrow$ CDP dose \\
\hline 14 & 22 & $\mathrm{~F}$ & $\begin{array}{l}\text { Hydrocephalus } \\
\text { R cerebellum } \\
\text { GTR }\end{array}$ & $\begin{array}{l}\text { M1 } \\
\text { T1 } \\
\text { Poor }\end{array}$ & 40 & Primary & $54+$ & $\begin{array}{l}\text { Persistent nausea } \\
\text { \& vomiting (2) } \\
\text { Tinnitus (2) } \\
\text { Neutropenia (2) } \\
\text { Thrombocytopenia (2) } \\
\text { Absent DTR (2) }\end{array}$ & CTX no dose change \\
\hline
\end{tabular}




\begin{tabular}{|c|c|c|c|c|c|c|c|c|c|}
\hline & Age & Sex & $\begin{array}{c}\text { Tumor location } \\
\& \text { surgery }\end{array}$ & $\begin{array}{l}\text { Chang } \\
\text { staging }\end{array}$ & $\begin{array}{l}\text { Relapse-free } \\
\text { survival (mo.) }\end{array}$ & $\begin{array}{l}\text { Relapse } \\
\text { site }\end{array}$ & $\begin{array}{c}\text { Overall } \\
\text { survival (mo.) }\end{array}$ & $\begin{array}{l}\text { Chemotherapy } \\
\text { toxicity }^{\mathrm{a}}\end{array}$ & $\begin{array}{l}\text { Chemotherapy } \\
\text { dose modification }\end{array}$ \\
\hline 15 & 36 & $M$ & $\begin{array}{l}\text { Hydrocephalus } \\
\text { Vermis } \\
\text { PR }\end{array}$ & $\begin{array}{l}\text { M3 } \\
\text { T1 } \\
\text { Poor }\end{array}$ & $19+$ & - & $19+$ & $\begin{array}{c}\text { Anemia (1) } \\
\text { Neutropenia (1) }\end{array}$ & \\
\hline 16 & 19 & $\mathrm{~F}$ & $\begin{array}{c}\text { Hydrocephalus } \\
\text { Vermis } \\
\text { L brainstem } \\
\text { STR }\end{array}$ & $\begin{array}{l}\text { M3 } \\
\text { T1 } \\
\text { Poor }\end{array}$ & $25+$ & - & $25+$ & $\begin{array}{c}\text { Anemia (1) } \\
\text { Neutropenia (1) }\end{array}$ & \\
\hline 17 & 33 & $M$ & $\begin{array}{l}\text { Hydrocephalus } \\
\text { 4th Ventricle } \\
\text { STR }\end{array}$ & $\begin{array}{l}\text { M2 } \\
\text { T1 } \\
\text { Poor }\end{array}$ & $96+$ & - & $96+$ & $\begin{array}{l}\text { Thrombocytopenia } \\
\text { (1) }\end{array}$ & \\
\hline
\end{tabular}

Abbreviations: cl, clearance; GTR, gross total resection; abd., abdominal; CDP, cisplatin; CTX, cyclophosphamide; D/C, discontinued; DTR, deep tendon reflexes; PR, <50\% resection; STR, $>50 \%$ resection; $\mathrm{RT}$, radiation therapy; VCR, vincristine.

${ }^{a}$ Number in parentheses is chemotherapy course when toxicity first appeared.

cycles of chemotherapy. No patient required transfusion; granulocyte colony-stimulating factor support was used in 1 patient during 3 cycles of chemotherapy. One patient required antibiotics for febrile neutropenia. Vincristine was discontinued in 7 patients after 1 to 6 cycles due to toxicity. One patient received no post-radiation VCR and another patient received VCR only intermittently during chemotherapy. On the POG protocol, 5 of 7 patients required chemotherapy dose reduction. Four patients had neutropenia $(<500 \mu \mathrm{l})$ on 14 cycles of chemotherapy, 3 patients thrombocytopenia $(<50,000 \mu \mathrm{l})$ on 14 cycles, and 3 (hemoglobin $<10 \mathrm{~g}$ ) anemia on 14 cycles of chemotherapy. No patient required transfusion and one patient required antibiotics for febrile neutropenia.

\section{Discussion}

In this study of 17 adult patients with infratentorial medulloblastoma, the median relapse-free survival for the entire cohort was 48 months, with no statistically significant difference between the 2 treatments. The POG patients' overall survival was not statistically different from that of the Packer patients' survival $(P$ value $=0.058)$, but it approached significance. Because of the small sample size, our data were inadequate to support any conclusions about the differences in relapse-free and overall survival between the 2 protocols. Both protocols included good and poor risk adult patients because in a previous pediatric trial poor-risk patients receiving the Packer regimen had a better outcome than did good-risk patients treated with radiation alone (Packer et al., 1991, 1994). Patients were considered poor risk if there was evidence of brainstem involvement, tumor filling the fourth ventricle extending into the foramina of Luschka or Magendiae producing internal hydrocephalus, or metastatic disease (Chang et al., 1969). For the pediatric trial, median progression-free 5-year relapse was $85 \%$ (Packer et al., 1994). In our study, there was no significant difference in the percentage of poor-risk patients on each treatment protocol or in the patients' relapse-free $(P=0.570)$ or overall survival $(P=0.161)$. The good- and poor-risk adult medulloblastoma patients appeared to have a significantly poorer outcome than the poor-risk pediatric patients with similar treatment. In contrast, a previous retrospective review of adult patients with medulloblastoma found 5-year relapse-free and overall survivals to be similar in adults and children (68\% and $58 \%)$, with a median survival of 72 months (Le et al., 1997).

Forty-seven percent of our patients had gross total resection, and $70.6 \%$ were poor risk, which is similar to data in 2 previous adult trials where gross total resection was carried out in $48 \%$ and $50 \%$ of patients in which $37 \%$ and $58 \%$ were poor risk (Frost et al., 1995; Prados et al., 1995). In the trial by Prados et al., 23 of 34 adults older than 15 years received chemotherapy, 5 of whom were treated with the Packer protocol. Patients receiving

Table 3. Chemotherapy treatment-related complications on 2 protocols studied

\begin{tabular}{lcc} 
Packer $(n=10)^{\mathrm{a}}$ & Complications & POG $(n=7)^{\mathrm{a}}$ \\
\hline 3 & Abdominal pain, persistent nausea, vomiting & 1 \\
5 & Peripheral neuropathy & 2 \\
7 & $>20$ db hearing loss/tinnitus & 3 \\
2 & $>25 \%$ decrease creatinine clearance & 0 \\
1 & Carbon monoxide lung diffusion capacity $\downarrow$ by $>40 \%$ & 0 \\
3 & Anemia Hgb $(<10 \mathrm{~g})$ & 3 \\
6 & Neutropenia $(<500 \mu \mathrm{l})$ & 4 \\
6 & Thrombocytopenia $(<50,000 \mu \mathrm{l})$ & 3 \\
\hline
\end{tabular}

Abbreviations: POG, Pediatric Oncology Group; db, decibel; Hgb, hemoglobin.

${ }^{a}$ Number of patients. 
adjuvant chemotherapy in this trial survived significantly longer than did those not receiving adjuvant chemotherapy. No discussion of toxicity by treatment group other than myelosuppression and a single patient with vincristine neuropathy is present in the Prados et al. study. In a subsequent study from the same institution over a similar time span, with presumably many overlapping patients, no difference in survival was found between patients treated with or without chemotherapy on univariate or multivariate analysis. In that study, the chemotherapy group had more poor-risk patients (Le et al., 1997). In a recent series of 42 childhood and 42 adult medulloblastoma patients, the tumors in adults had significantly higher median pCNA and MIB-1 labeling indices, which might explain a poorer survival for adults (Giordana et al., 1997). It is always difficult to compare patients treated at multiple institutions and given different treatments.

The toxicity of the Packer protocol appears to be much greater in adults than in children. Sixty of 63 patients $(95 \%)$ in the childhood study received full-dose CDP until the fifth cycle of treatment, and $44 \%$ received the full total dose of CDP. In our study, only $30 \%$ of adult patients received full-dose CDP by the sixth cycle, and no patient received full-dose CDP on all 8 cycles. Furthermore, in the study of Packer et al., 1994, 14.2\% of children required a dose reduction of CCNU due to myelotoxicity, whereas $100 \%$ of adult patients treated similarly required a CCNU dose reduction. In addition, one adult patient on the Packer protocol had a $>40 \%$ decrease in diffusion capacity. The VCR dose was decreased in $25 \%$ of children treated on the Packer protocol compared with $90 \%$ of our adult patients in which 6 of 10 adults received less than 3 cycles of vincristine. Toxicity was substantially less in patients treated with the POG regimen, but a meaningful Q TWIST analysis comparing the quality of life on the two protocols could not be completed because of small sample size.

In summary, the role of multiagent adjuvant chemotherapy in the treatment of good- and poor-risk adults with either the Packer or POG regimen requires further study and a larger clinical randomized trial. Adequate assessment of statistical differences between the median relapse-free and overall survival in the protocols is not possible in the present study. The relapse-free and overall survival rate appears lower in adults than in children. Adults with medulloblastoma treated on the Packer protocol experienced considerably greater chemotherapy-related toxicity than did children treated on an identical protocol. We have not demonstrated that chemotherapy is effective when added to craniospinal radiation in adult patients with medulloblastoma. A prospective multi-institutional randomized clinical trial is needed to answer the question.

\section{References}

Aragones, M.P., Magallon, R., Piqueras, C., Ley, L., Vaquero, J., and Bravo, G. (1994) Medulloblastoma in adulthood: Prognostic factors influencing survival and recurrence. Acta Neurochir. (Wien) 127, 65-68.

Biegel, J.A., Rorke, L.B., Packer, R.J., Sutton, L.N., Schut, L., Bonner, K., and Emanuel, B.S. (1989) Isochromosome $17 q$ in primitive neuroectodermal tumors of the central nervous system. Genes Chromosomes Cancer 1, 139-147.

Bloom, H.J.G., and Bessell, E.M. (1990) Medulloblastoma in adults: A review of 47 patients between 1952 and 1981. Int. J. Radiat. Oncol. Biol. Phys. 18, 763-772.

Chang, C.H., Housepian, E.M., and Herbert, C., Jr. (1969) An operative staging system and a megavoltage radiotherapeutic technic for cerebellar medulloblastomas. Radiology 93, 1351-1359.

Duffner, P.K., Horowitz, M.E., Krischer, J.P., Friedman, H.S., Burger, P.C., Cohen M.E., Sanford, R.A., Mulhern, R.K., James, H.E., Freeman, C.R., Seidel, F.G., and Kun, L.E. (1993) Postoperative chemotherapy and delayed radiation in children less than three years of age with malignant brain tumors. New Engl. J. Med. 328, 1725-1731.

Frost, P.J., Laperriere, N.J., Wong, C.S., Milosevic, M.F., Simpson, W.J.S., and Pintilie, M. (1995) Medulloblastoma in adults. Int. J. Radiat. Oncol. Biol. Phys. 32, 951-957

Giordana, M.T., Cavalla, P., Dutto, A., Borsotti, L., Chiò, A., and Schiffer, D. (1997) Is medulloblastoma the same tumor in children and adults? J. Neurooncol. 35, 169-176.

Hubbard, J.L., Scheithauser, B.W., Kispert, D.B., Carpenter, S.M., Wick, M.R., and Laws, E.R., Jr. (1989) Adult cerebellar medulloblastomas: The pathological, radiographic, and clinical disease spectrum. J. Neurosurg. 70, 536-544.
Kopelson, G., Linggood, R.M., and Kleinman, G.M. (1982) Medulloblastoma in adults: Improved survival with supervoltage radiation therapy. Cancer 49, 1334-1337.

Le, Q.T., Weil, M.D., Wara, W.M., Lamborn, K.G., Prados, M.D., Edwards, M.S.B., and Gutin, P.H. (1997) Adult medulloblastoma: An analysis of survival and prognostic factors. Cancer J. Sci. Am. 3, 238-245.

MacDonald, D.R., Cascino, T.L., Schold, S.C., Jr., and Cairncross, J.G. (1990) Response criteria for phase II studies of supratentorial malignant glioma. J. Clin. Oncol. 8, 1277-1280.

Packer, R.J., Siegel, K.R., Sutton, L.N., Litmann, P., Bruce, D.A., and Schut, L. (1985) Leptomeningeal dissemination of primary central nervous system tumors of childhood. Ann. Neurol. 18, 217-221.

Packer, R.J., Sutton, L.N., Goldwein, J.W., Perilongo, G., Bunin, G., Ryan, J., Cohen, B.H., D'Angio, G., Kramer, E.D., Zimmerman, R.A., Rorke, L.B., Evans, A.E., and Schut, L. (1991) Improved survival with the use of adjuvant chemotherapy in the treatment of medulloblastoma. J. Neurosurg. 74, 433440 .

Packer, R.J., Sutton, L.N., Elterman, R., Lange, B., Goldwein, J., Nicholson, H.S., Mulne, L., Boyett, J., D'Angio, G., and Wechsler-Jentzsch, K., Reaman, G., Cohen, B.H., Bruce, D.A., Rorke, L.B., Molloy, P., Ryan, J., LaFond, D., Evans, A.E., and Schut, L. (1994) Outcome for children with medulloblastoma treated with radiation and cisplatin, CCNU, and vincristine chemotherapy. J. Neurosurg. 81, 690-698.

Prados, M.D., Warnick, R.E., Wara, W.M., Larson, D.A., Lamborn, K., and Wilson, C.B. (1995) Medulloblastomain adults. Int. J. Radiat. Oncol. Biol. Phys. 32, 1145-1152. 